\title{
A case of methotrexate resistant gestational trophoblastic neoplasia
}

\author{
Sanjay Singh*, Akhileshwar Singh, Shakti Vardhan
}

Department of Obstetrics and Gynecology, Armed Forces Medical College, Pune, Maharashtra, India

Received: 25 September 2018

Accepted: 22 October 2018

\author{
*Correspondence: \\ Dr. Sanjay Singh, \\ E-mail: drsanjaysingh@gmail.com
}

Copyright: (c) the author(s), publisher and licensee Medip Academy. This is an open-access article distributed under the terms of the Creative Commons Attribution Non-Commercial License, which permits unrestricted non-commercial use, distribution, and reproduction in any medium, provided the original work is properly cited.

\begin{abstract}
Gestational trophoblastic neoplasia (GTN) is a subset of gestational trophoblastic disease (GTD) which has a propensity to invade locally and metastasize. Patients with low risk GTN generally respond well to single agent chemotherapy (methotrexate (MTX) or actinomycin-D (ACT-D). However, high risk cases may develop resistance or may not respond to this first-line chemotherapy and are unlikely to be cured with single-agent therapy. Therefore, combination chemotherapy is used for treatment of these cases. Here we present a 25 years old P2 L2 A1 lady, who was initially treated at a peripheral hospital with multiple doses of Injection methotrexate with a working diagnosis of persistent trophoblastic disease. She didn't respond to this treatment and reported to our centre for further management. On evaluation she was found to be a case of high risk GTN (invasive mole) (I:8) for which she was put on combination chemotherapy in the form of Etoposide-Methotrexate-Actinomycin-Cyclophosphamide-Oncovin (EMA-CO) regime. She responded to this treatment and is presently asymptomatic and is under regular follow up.
\end{abstract}

Keywords: Abnormal uterine bleeding, b-hCG, EMA-CO, Gestational trophoblastic neoplasia, Invasive mole

\section{INTRODUCTION}

Gestational Trophoblastic Neoplasia is a subset of GTD which despite having high propensity to invade surrounding structures and to metastasize, responds very well to chemotherapy. ${ }^{1}$ This is one of few neoplasias where chemotherapy can be started even without a tissue diagnosis.

Patients, who respond poorly to single agent chemotherapy, should be given second line combination chemotherapy with or without surgery without much delay. High remission rates have been reported with the treatment. Counselling and strict follow up with beta HCG levels form strong pillars of management of this disease. $^{2}$

Here authors present a case of 25 years old P2 L2 A1 lady, who was initially treated at a peripheral hospital with multiple doses of Injection methotrexate with a working diagnosis of persistent trophoblastic disease. As she didn't respond to this treatment she reported to our centre where on evaluation she was found to have high risk GTN (invasive mole) (I:8). She was treated with combination chemotherapy (EMA-CO). She responded to this treatment and is presently asymptomatic and is under regular follow up.

\section{CASE REPORT}

25 years old P2 L2 A1 lady presented to our institute with complaints of on and off heavy menstrual bleeding for past 12 months following suction and evacuation done for missed abortion at a primary health care centre.

Products were not sent for histopathological examination. She also complained of lethargy and weakness for 2 months. However, there was no history of syncopal attacks. She also complained of cough and breathlessness for 1 month. There was no history suggestive of hyperthyroidism. There was no complaint of any lump or pain abdomen, discharge per vaginum, haemoptysis and 
motor or sensory deficits. There was no history suggestive of raised intracranial tension in the form of headache, vomiting, visual disturbances or focal deficits. She was being treated at the peripheral health care centre with varying doses of methotrexate for past one month. Basis of starting this treatment in the peripheral hospital was ultrasound finding of multiple cystic dilated vascular spaces seen in uterus and blood for beta HCG levels $24000 \mathrm{mIU} / \mathrm{mL}$.

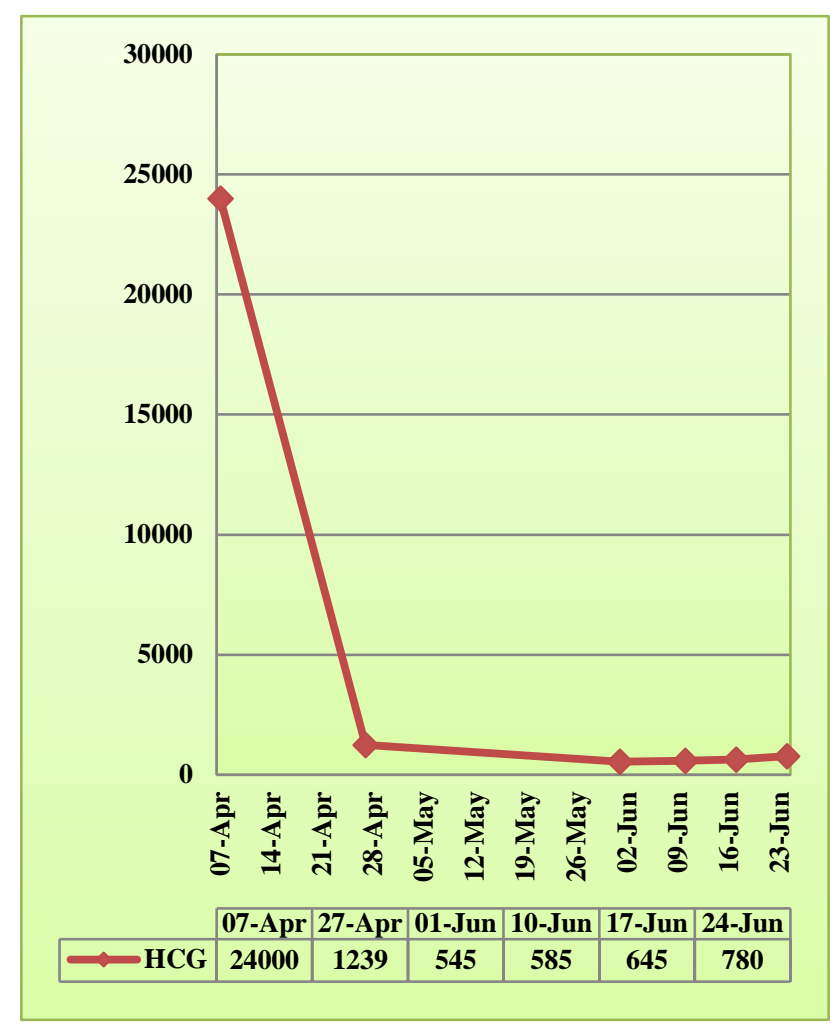

Figure 1: Log curve of b-hCG, while undergoing treatment at peripheral hospital. Last three values done at our institute at weekly interval shows rising trend.

Initially she was given a course of 4 doses of Injection methotrexate $50 \mathrm{mg}$ intramuscularly on alternate days. As b-hCG value was not reducing satisfactorily she was given another course of 2 doses of Injection methotrexate $60 \mathrm{mg}$ intravenous weekly for 2 weeks after one month of interval from the first course. However, in spite of this treatment at the peripheral hospital, she continued to be symptomatic and reported to us. On examination at our centre, she was found to have mild pallor. Her BMI was $17.6 \mathrm{Kg} / \mathrm{m}^{2}$. Respiratory system, central nervous system and cardiovascular system examination were essentially normal. Per abdominal examination revealed a 14-16 weeks size extremely soft mass (probably uterus) which was mildly tender with no organomegaly or free fluid in abdomen. Per speculum examination showed altered blood at external os but no active bleeding.

On per vaginal examination uterus was 16 weeks size, soft and tender. Bilateral adnexae were free and there was no forniceal tenderness. After history and examination, pregnancy related complications, abnormal uterine bleeding and gestational trophoblastic disease were all considered as differential diagnoses. She was then thoroughly evaluated. Her haemoglobin was $9 \mathrm{gm} \%$. Biochemical parameters including renal and liver function tests were within normal limits. Blood group was 'A' positive. TSH was $2.32 \mathrm{uIU} / \mathrm{mL}$. Log curve of b$\mathrm{hCG}$, while undergoing treatment at peripheral hospital is shown in Figure 1.

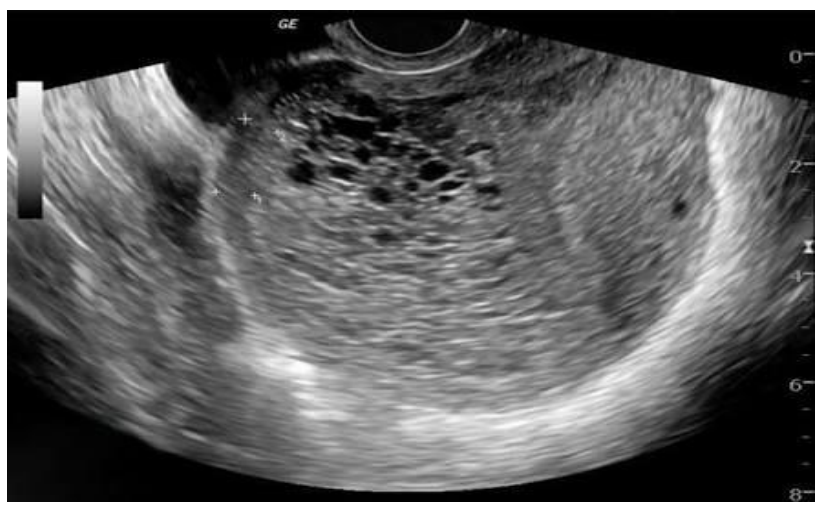

Figure 2: Ultrasound pelvis revealing a bulky uterus (size $14 \mathrm{~cm} \times 12 \mathrm{~cm} \times 9 \mathrm{~cm}$ ) with heterogenous mass measuring $7 \mathrm{~cm} \mathrm{X} 5 \mathrm{~cm} \mathrm{X} 4.5 \mathrm{~cm}$ near fundus with multiple cystic vascular spaces. Endo-myometrial junction not well delineated.

As can be seen, beta HCG with treatment had fallen from $24000 \mathrm{mIU} / \mathrm{mL}$ to $1200 \mathrm{mIU} / \mathrm{mL}$ and subsequently to $545 \mathrm{mIU} / \mathrm{mL}$. However, 3 serial serum b-hCG levels done at weekly interval at our centre suggested a rising trend (585mIU/ml, 645mIU/ml, and $780 \mathrm{mIU} / \mathrm{ml})$. Ultrasound pelvis done at our institute revealed a bulky uterus (size $14 \mathrm{~cm} \mathrm{X} 12 \mathrm{~cm} \mathrm{X} \mathrm{9cm)} \mathrm{with} \mathrm{heterogenous} \mathrm{mass}$ measuring $7 \mathrm{~cm} \mathrm{X} 5 \mathrm{~cm} \mathrm{X} 4.5 \mathrm{~cm}$ near fundus with multiple cystic vascular spaces (Figure 2).

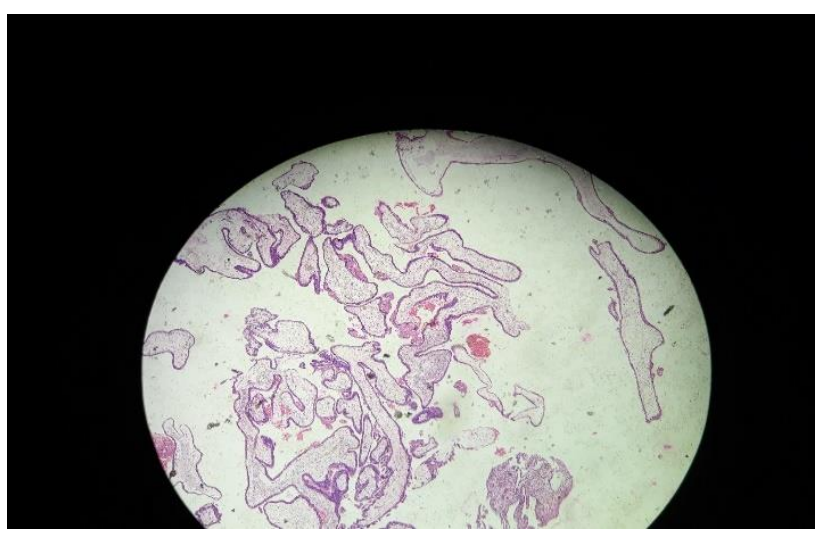

Figure 3: Histopathology revealing decidual tissue with numerous chorionic villi with cistern spaces and hydropic changes.

Endo-myometrial junction was not well delineated. There were no adnexal masses; intra-uterine of extra-uterine 
sacs. Our plan to manage her included a tissue diagnosis. Histopathology revealed decidual tissue with numerous chorionic villi with cistern spaces and hydropic changes (Figure 3).

It was then decided to further evaluate her for Gestational Trophoblastic Neoplasia. MRI abdomen and pelvis showed an invasive mass in uterus involving serosa but not involving adnexae, pouch of douglas, any other pelvic or extra pelvic tissues. Possibility of Invasive Mole or persistent Gestational Trophoblastic Disease was suggested. Final diagnosis of Invasive Mole / persistent Gestational Trophoblastic Disease with resistance to single agent chemotherapy or suboptimal therapy was made. The staging of the disease was done as per International Federation of Gynecology and Obstetrics (FIGO) (2009) staging (stage 1) along with prognostication of the disease by WHO scoring system (high risk; score 8), and treatment was started with multidrug chemotherapy in the form of EMA-CO regime. 3 After 6 weeks of treatment, her beta HCG levels had fallen to $15 \mathrm{mIU} / \mathrm{mL}$. Uterine size reduced significantly, and clinical improvement was also evident. She is presently being followed up with serial beta HCG levels. Appropriate contraception for a period not less than 24 months in the form of an oral contraceptive pill has been advised. She also has been advised to come for preconceptional counseling, in case she plans to conceive.

\section{DISCUSSION}

Gestational Trophoblastic Disease (GTD) is a group of disorders related to abnormal placental trophoblastic proliferation. Incidence of GTD ranges from 1-3 per thousand live births which vary across different regions of India, maximum being in north India and on the lower side in southern India. Its incidence is 1 per 1000 live births in the USA. ${ }^{4}$ GTN is a subset of GTD which fails to resolve spontaneously or with standard medication and tend to invade uterine wall or metastasize, as can be noted in our case. This includes Invasive mole, Choriocarcinoma, Placental site trophoblastic tumour and Epithelioid trophoblastic tumours. 50\% of GTN develop following $\mathrm{H}$ Mole, $25 \%$ follow miscarriage or tubal pregnancy, and another $25 \%$ develop after term or preterm pregnancy. ${ }^{5}$ Our case occurred following a case of molar pregnancy. All these four tumours are though histologically distinct, they are generally diagnosed by persistently rising/ plateauing of serum b-hCG levels. This is because representative tissue is frequently not available for histopathological study. In our case, three weekly b-hCG values kept on rising and histopathological study confirmed the intact morphological feature of chorionic villi.

Hydatidiform moles are generally benign, but one or more of following risk factors make them more prone to develop trophoblastic neoplasia. Most importantly, complete mole comparing partial mole carries more risk of developing GTN (15-20\% Vs 1-5\%). Other risk factors include: pregnancy before 20 years or after 35 years of age, very high levels of b-hCG levels $(>1,00,000$ $\mathrm{mIU} / \mathrm{mL}$ ), large tumour size, theca-lutein cysts larger than $6 \mathrm{cms}$, and slow decline in b-hCG levels. ${ }^{6,7}$ Risk factors present in our case were large tumour size and rising b-hCG levels. Generally, cases of GTNs present with abnormal vaginal bleeding (continuous/intermittent/sometimes massive haemorrhage) with sub involution of the uterus. Our case had on and off heavy vaginal bleeding and subinvlution of the uterus. If trophoblastic growth perforates myometrium, it may lead to intraperitoneal haemorrhage. Lower genital tract and distant metastasis may be present.

To diagnose GTN it is important to keep this entity as a possibility in cases of persistent abnormal vaginal bleeding following any type of pregnancy. It further entails measurement of blood b-hCG and diagnostic curettage. Lower genital tract metastasis and uterine size may be assessed during curettage. Biopsy for tissue diagnosis may lead to profuse bleeding and is considered unnecessary. After confirmation of the diagnosis and along with baseline b-hCG and hemogram assessment, assessment of local and distant spread of the disease is required to be done. For this one needs to do liver and renal function test, sonography, chest X-Ray/CT scan, brain and abdominopelvic CT scan or MR imaging. We followed this protocol of evaluation in our case. PET scan is occasionally required to find out mets.

Anatomical staging of GTN is done using International Federation of Gynecology and Obstetrics staging and diagnostic scoring system. ${ }^{8}$ This also includes a modification of World Health Organization (1983) prognostic scoring system. In this a score ranging from 0 to 4 are given for each of several categories included in it. Those with WHO scores 0-6 are considered to have lowrisk disease and those with a score $\geq 7$ are considered in the high-risk group. Our patient belonged to Stage/Group I:8. Invasive moles are the most common GTN and almost all arise from complete or partial moles. ${ }^{9}$ Characteristically the villous structure is preserved in them and they are capable of invading not only the myometrium (as can be seen in our case) but also the peritoneum, adjacent parametrium and vaginal vault. They are however less prone to metastasize.

GTN cases are best managed by oncologists. We managed our case in consultation with medical and surgical oncologist. Chemotherapy is usually the treatment. Single agent chemotherapy protocols in form of either methotrexate (less toxic) or actinomycin-D are usually considered adequate for non-metastatic or lowrisk metastatic neoplasia. ${ }^{10}$ Combination chemotherapy (e.g., EMA-CO: etoposide, methotrxate, actinomycin-D, cyclophosphomide and oncovin (vincristine), is used for high-risk disease (as was our case) and its cure rate has been reported to $90 \% .^{11}$ Adjuvant surgical and radiotherapy may also be used as per need. ${ }^{12}$ 
As per American College of Obstetricians and Gynecologists, 2014, repeat dilatation and curettage increases morbidity and mortality of the patients by increasing risk of uterine perforation, haemorrhage, infection, uterine adhesion and anaesthetic complications and hence is not recommended. However, repeat uterine curettage is standard practise for post molar GTN management in Europe. This is done in an effort to avoid or minimize chemotherapy. ${ }^{13}$ In our case we did repeat curettage for the tissue diagnosis. Low risk GTN cases are monitored with weekly b-hCG measurements until with treatment b-hCG levels are undetectable for 3 consecutive weeks. This is followed by monthly assessment of b-hCG levels for 12 months. However, patients with high risk disease as in our case are followed for 24 months due to greater risk of late relapse. Our patient is still under follow up. Effective contraception is of utmost importance during this period to avoid teratogenic accidental exposure of the drug to the foetus and to avoid confusion from rising b-hCG levels caused by accidental superimposed pregnancy. We put our patient on OCPs. Though treated patients of GTN don't become infertile there is a $2 \%$ risk for developing trophoblastic disease in the subsequent pregnancy. ${ }^{14}$ For this sonography is recommended at early duration of pregnancy as well at later date also if indicated.

\section{CONCLUSION}

Patients with low risk GTN generally respond well to single agent methotrexate. However, high risk cases like in our case may develop resistance or may not respond to this first-line chemotherapy. Alternative combination chemotherapy leads to complete remission in these patients as can be seen in our case.

\section{ACKNOWLEDGMENTS}

Authors would like to thank Dr. Nirupam, Dr. Khanna for his support during study.

Funding: No funding sources Conflict of interest: None declared

Ethical approval: Not Required

\section{REFERENCES}

1. Berkowitz RS, Goldstein DP. Current management of gestational trophoblastic diseases. Gynecol Oncol. 2009;112(3):654-62.

2. Seckl MJ, Sebire NJ, Berkowitz RS. Gestational trophoblastic disease. Lancet. 2010;376(9742):71729.
3. Alazzam M, Tidy J, Osborne R, Coleman R, Hancock BW, Lawrie TA. Chemotherapy for resistant or recurrent gestational trophoblastic neoplasia. Cochrane Database Syst Rev. 2016;1:CD008891

4. Ballal PK, Kushtagi PS, Nikil PR. Variation in Prevalence of Gestational Trophoblastic Disease in India. Indian J Gynecol Oncol.2016;14 (3):1-5.

5. Goldstein DP, Berkowitz RS: Current management of Gestational trophoblastic neoplasia. Hematol Oncol Clin North Am.2012;26(1):111.

6. Kang WD, Choi HS, Kim SM: Prediction of persistent gestational trophoblastic neoplasia: the role of hCG level and ratio in 2 weeks after evacuation of complete mole. Gynecol Oncol 2012;124(2):250.

7. Altieri A, Franceschi S, Ferlay J, Smith J, La Vecchia C. Lancet Oncol. 2003; 4(11):670-8.

8. FIGO Committee on Gynecologic Oncology. Current FIGO staging for cancer of the vagina, fallopian tube, ovary and gestational trophoblastic neoplasia. Int J Gynaecol Obstet 2009;105(1):3-4.

9. Sebire NJ, Lindsay I, Fisher RA: Over diagnosis of complete and partial hydatidiform mole in tubal ectopic pregnancies. Int $\mathrm{J}$ Gynecol Pathol. 2005;24(3):260.

10. Horowitz NS, Goldstein DP, Berkowitz RS: Management of gestational trophoblastic neoplasia. Semin Oncol.2009;36(2):181.

11. Lurain JR. Gestational trophoblastic disease I; epidemiology, pathology, clinical presentation, and diagnosis of gestational trophoblastic disease, and management of hydatidiform mole. Am J Obstet Gynecol.2010;203(6):531.

12. Hanna RK, Soper JT. The role of surgery and radiation therapy in the management of gestational trophoblastic disease. Oncol.2010;15(6):593.

13. van Trommel NE, Massuger LF, Verheijen RH, Sweep FC, Thomas CM. The curative effect of a second curettage in persistent trophoblastic disease: a retrospective cohort survey. Gynecologic Oncol. 2005;99(1):6-13.

14. Garrett LA, Garner EI, Feltmate CM, Goldstein DP, Berkowitz RS. Subsequent pregnancy outcomes in patients with molar pregnancy and persistent gestational trophoblastic neoplasia. Obstet Gynecol Surv. 2008;63(11):704-5.

Cite this article as: Singh S, Singh A, Vardhan S. A case of methotrexate resistant gestational trophoblastic neoplasia. Int J Reprod Contracept Obstet Gynecol 2018;7:5178-81. 